\title{
Symposium summary
}

\author{
Sue C. Kaste
}

Received: 30 October 2008 / Accepted: 3 November 2008 / Published online: 16 December 2008

(C) Springer-Verlag 2008

Keywords ALARA P Pediatric oncology

The ALARA concept in pediatric imaging emphasizes control of exposure of children to ionizing radiation from medical imaging. While this concept is very important for all pediatric patients, it takes on a particular-and

Dr. Kaste has no relevant financial relationships or potential conflicts of interest related to the material to be presented.

\section{S. C. Kaste $(\bowtie)$}

Division of Diagnostic Imaging,

Department of Radiological Sciences,

St. Jude Children's Research Hospital MSN \#220,

262 Danny Thomas Place,

Memphis, TN 38105-3678, USA

e-mail: sue.kaste@stjude.org

\section{S. C. Kaste}

Department of Oncology, St. Jude Children's Research Hospital,

Memphis, TN, USA

\section{S. C. Kaste}

Department of Radiology,

University of Tennessee Health Science Center,

Memphis, TN, USA challenging - role in the care of children and adolescents who require repeated and long-term imaging. Such is the case of pediatric patients undergoing evaluation and treatment for malignancies and for whom repeated exposures to ionizing radiation may enhance risks for developing new cancers.

Today's symposium addressed many of the issues in providing optimal care and imaging in pediatric oncology while considering ways to minimize patient exposures to ionizing radiation. The world-renowned speakers participating today are leaders in their respective fields and agree with the need to further refine imaging frequency and techniques according to ALARA principles. Of particular note is the need for the Society for Pediatric Radiology (SPR) and the Children's Oncology Group (COG) to work more closely together to optimize imaging for clinical and research initiatives. With the rapid advancement of technologic development, organizations such as SPR and COG, dedicated to the care and well-being of children, should spearhead defining roles for, techniques of, and incorporation of new modalities into the armamentarium of pediatric imaging. Through such collaborative initiatives, progress in patient care is enhanced. 